\title{
A Guiding Nightlight Decreases Fear of Falling and Increases Sleep Quality of Community-Dwelling Older People: A Quantitative and Qualitative Evaluation
}

\author{
Thessa W. Thölking ${ }^{a, b}$ Eef C.T. Lamers ${ }^{b}$ Marcel G.M. Olde Rikkert ${ }^{a}$ \\ a Department of Geriatric Medicine, Radboud University Medical Center, Nijmegen, The Netherlands; \\ ${ }^{b}$ Gight-BV, Nijmegen, The Netherlands
}

\section{Keywords}

Falls · Night · Guiding light · Fear of falling · Prevention

\begin{abstract}
Background: Even though poor lighting at nighttime is an important risk factor for falls (and most falls occur during the night), lighting interventions to improve nightly lighting from bed to bathroom are rarely evaluated for fall prevention. Objective: We tested the hypothesis that an automated guiding light would reduce nightly fear of falling (FOF) and increase sleep quality of community-dwelling older people. Methods: This study had a pragmatic uncontrolled beforeafter design, including participants during a period of 8 months if they (i) were aged at least 65 years, (ii) ambulated independently at night, and (iii) had no cognitive or audiovisual impairments obstructing outcome measurement. Automated LED strips (Gight ${ }^{\mathrm{TM}}$ ) were installed in the participants' homes. The primary outcome measure was overnight FOF on a scale of 0-10. Secondary outcome measures included sleep quality on a scale of $0-10$ and fall rate. Additionally, a sample of participants was interviewed about their experiences with Gight. Results: Sixty-four participants were included (mean age: $80.8 \pm 8.1$ years; $89 \%$ living independently). Mean study length was 118 days (range: $30-231$ ). In the
\end{abstract}

karger@karger.com www.karger.com/ger

Karger ${ }^{\prime}$ '

GOPEN ACCESS
(C) 2020 The Author(s)

Published by S. Karger AG, Basel

This is an Open Access article licensed under the Creative Commons Attribution-NonCommercial-4.0 International License (CC BY-NC) (http://www.karger.com/Services/OpenAccessLicense), applicable to the online version of the article only. Usage and distribution for commercial purposes requires written permission. intention-to-treat analysis, overnight FOF declined from $5.5 \pm 3.0$ to $3.8 \pm 3.2(p=0.001)$, and sleep quality increased from $6.7 \pm 2.4$ to $7.4 \pm 1.7(p=0.012)$. The fall rate during the study was too low to detect changes. Participants appreciated Gight ( $8.4 \pm 0.8$ on a scale of 10$)$, and the majority (57\%) reported a subjective decrease in FOF. Conclusion: Gight shows promising results for overnight FOF and sleep quality, but the effect of lighting interventions on fall rate should be evaluated further before widespread implementation.

(C) 2020 The Author(s)

Published by S. Karger AG, Basel

\section{Introduction}

Falling is a major risk to the health of older people. In the Netherlands, 102,000 people over the age of 65 years visited the emergency department because of fall-related injuries in 2017 [1]. Approximately one third of the community-dwelling older persons falls at least once a year [2]. These alarming numbers are expected to rise globally with societal aging, substantially increasing personal, societal, and financial impact.

Previous research has shown that insufficient lighting is a relevant risk factor for falls [3-5]. Similarly, Magota et al. [6] found that the majority of falls in a hospital set- 
ting occurs during the night. As vision significantly contributes to posture control and navigation, adequate lighting is of importance especially to older persons with peripheral sensory impairments [7-9]. In addition, agerelated decline in light passed through to the retina, and in the sensitivity of the retina itself, causes a need for higher light intensities to perform visually mediated tasks [8].

Ambulation towards the bathroom seems to be the nightly task causing the greatest fall risk. Research focusing on hospital incident reports noted that the majority of falls were related to toileting [6]. Similarly, nocturia has been identified as a risk factor for falls [10]. Since it has been reported that approximately $60 \%$ of older people toilet at least twice a night [11], lighting interventions during ambulation from bed to bathroom could minimize fall risk at night. Nevertheless, only limited research has been conducted with regard to automated lighting interventions for community-dwelling older people.

In this study, we aim to investigate the effect of installing a movement-driven, automated LED strip at ground level in the path from bed to bathroom on fear of falling (FOF), sleep quality, and fall rate.

\section{Materials and Methods}

\section{Design}

This study was set up as a pragmatic uncontrolled before-after study. Suitable applicants were included when they presented themselves as interested in the study from October 2017 onwards and remained in the study until its conclusion in June 2018. Therefore, study length varied for participants, with a maximum duration of 8 months.

\section{Participants}

The study was carried out with clients of the elderly care organization "BrabantZorg" in Oss (Netherlands), and due to slow inclusion rates, we also included other older people in Oss and surroundings at a later stage. Inclusion criteria were: (i) age $\geq 65$ years, (ii) toileting independently at night, and (iii) ability to generate the outcomes. Older people with severe cognitive, hearing, or vision impairments which impeded (paper and pen or interview guided) outcome registration were excluded from participation. Participants were recruited mainly after being informed by professionals from BrabantZorg, BrabantWonen, and the municipality of Oss, but also by Facebook posts, flyers, local paper advertisements, and promotion events in Oss.

\section{Intervention}

The investigated intervention was Gight ${ }^{\mathrm{TM}}$, an innovative automated guiding light. Gight consists of an in length adjustable LED strip $(12 \mathrm{~V})$ attached to the baseboard, activated by a motion sensor, causing it to light up for $30 \mathrm{~s}$ after motion. In this study, 1 long

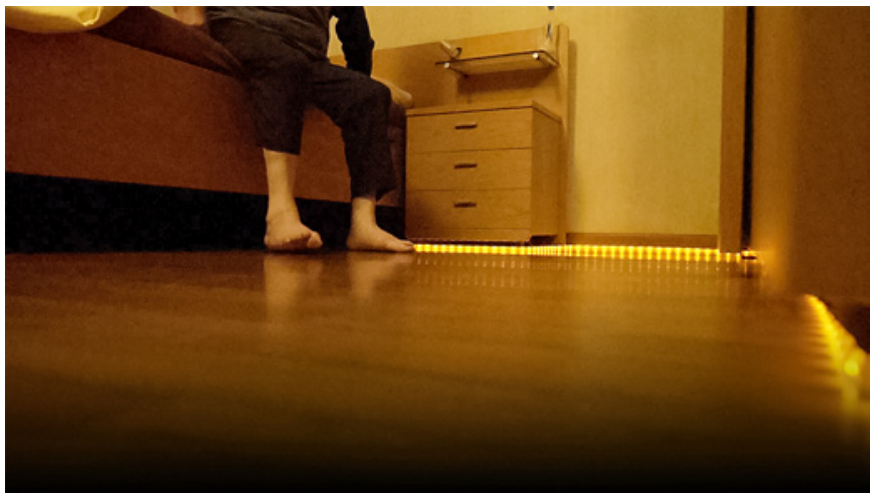

Fig. 1. Example of the Gight ${ }^{\mathrm{TM}}$ LED strip setup in a bedroom. The LED strip is activated by its motion sensor as the older person gets up from the bed.

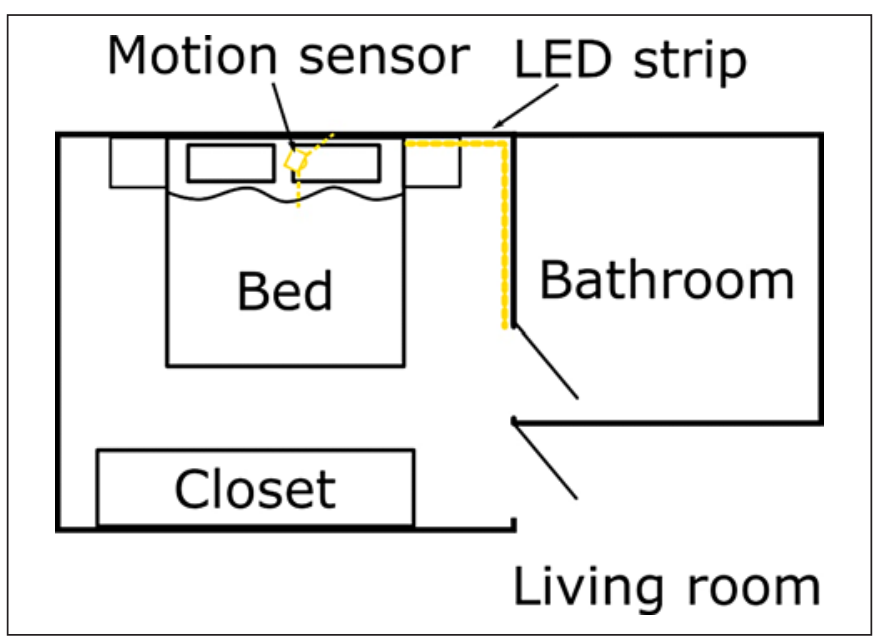

Fig. 2. Diagram of the Gight ${ }^{\mathrm{TM}}$ motion sensor and LED strip example setup in the bedroom displayed in Figure 1. It shows the motion sensor which is positioned under the bed and its detection range of $120^{\circ}$ and the LED strip. Motion sensor position and LED strip position and length were adapted to each participant's accommodation and wishes.

LED strip was installed in a room on the path generally walked from bed to bathroom. An example setup is shown in Figures 1, 2. Gight was installed at baseline measurement and kept until the conclusion of the study.

\section{Outcome Measures}

The primary outcome measure of this study was the monthly measured single-item overnight FOF on a scale from 0 to 10. It was measured by answering the question (in Dutch): "How concerned are you about the possibility of falling whilst getting out of bed at night to void? $0=$ not concerned at all, $10=$ very concerned." This item is adapted from the international version of the Falls Efficacy Scale (FES-I), which has no item on FOF overnight [12].

Secondary outcome measures were single-item sleep quality (range: 0-10), self-reported fall rate (represented as a fall/month 
fraction and as a dichotomous fall incidence), complete FES-I (range: 16-64), Pittsburgh Sleep Quality Inventory (PSQI) (range: 0-21), and the Older Persons and Informal Caregivers SurveyComposite Endpoint (TOPICS-CEP), which combines well-validated quality of life and functional performance scores (range: $0-10)$ [12-15]. Single-item sleep quality was measured monthly by a (Dutch) item of the PSQI: "During the past month, how would you rate your sleep quality overall? 0 = very bad, 10 = very good" [13]. At baseline measurement, participants reported their fall rate during the last 6 months. During the study, fall rate was registered on a fall calendar by participants, which was collected monthly. FES-I, PSQI, and TOPICS were administered at baseline before installation of Gight and as final measurement just before optional removal at the end of the study. TOPICS was also used to acquire descriptive variables, calculate frailty indices, and generate a combined quality of life $[16,17]$.

In addition, at the end of the study, qualitative interviews were held about the intervention with participants varying as much as possible in age, sex, fall rate, education level, living situation, and participation period until saturation of answers was reached. The interview consisted of 8 questions ( 5 open-ended, 2 semi-closedended, and 1 scale question) about the experienced effect, experienced value, and product features of Gight.

\section{Analytical Methods}

Statistical analysis consisted of an intention-to-treat (ITT) analysis, in which the last observation carried forward (LOCF) was treated as a final measurement in case of missing data, and a perprotocol (PP) analysis, in which only data from participants of whom a final measurement was obtained was analyzed. Outcome measures at baseline or final measurement (or LOCF) were analyzed with Wilcoxon signed-rank tests and paired $t$ tests for nonnormally and normally distributed data, respectively. Effect size $r$ was defined as $r=$ Wilcoxon $Z / \sqrt{ }(N)$. The $\chi^{2}$ test was used to analyze dichotomous fall incidence.

To determine the development of the single-item overnight FOF and sleep quality over time, these outcomes were calculated for every 30-day interval and compared with each other with Wilcoxon signed-rank tests. To investigate potential effect modifiers, Pearson correlations and multivariate regressions were conducted for change in single-item overnight FOF and sleep quality, and 4 independent variables were chosen in advance: measurement at baseline (FOF or sleep quality, respectively), frailty index, living situation (alone yes/no), and time of Gight use (days). The dependent variable in both regression analyses was the difference between the single-item FOF score or sleep quality score at the end of the study (if needed complemented by LOCF values) and baseline ( $\Delta=$ value at LOCF - baseline). All quantitative data of the study were analyzed using IBM SPSS Statistics 23.

All qualitative interviews were recorded, and summaries of the interviews were constructed from these recordings. A first researcher (T.W.T.) derived a code list from these summaries, upon which the frequency of codes in participants' answers to openended and semi-closed-ended questions were determined. A second researcher (E.C.T.L.) checked the code list, and in case of different interpretations, consensus was sought together with the first researcher. For the scale question, mean and standard deviation were calculated.

Evaluation Study of a Guiding Nightlight for Older People

\section{Results}

\section{Participants}

Sixty-four participants were included in the study, with a mean participation length of $118 \pm 57$ days. Forty-four (69\%) participants completed the study, with an average of $129 \pm 55$ days of participation, and $6(9 \%)$ participants withdrew from the study before the first follow-up visit. Total loss to follow-up $(n=20)$ was caused by death $(n=7)$, lack of satisfaction with the product $(n=4)$, move or admission to a care facility $(n=3)$, unsuitable living accommodation $(n=2)$, and other reasons $(n=4)$.

\section{Baseline Data}

Participants' mean age was $81 \pm 8$ years. Two participants (64 and 63 years of age) did not meet the inclusion criterion of age but were included as their living situation in a retirement community and retirement home, respectively, still placed them in the target population of this study. Forty-two (66\%) participants were female, and another $42(66 \%)$ participants lived independently alone, whilst 15 (23\%) lived independently with others, and 7 (11\%) lived in a retirement or nursing home. The mean frailty index of participants was $0.29 \pm 0.13$, and 38 (59\%) participants reported 3 or more (chronic) conditions in the last year. Additional demographic variables are included in Table 1.

\section{Intervention}

Gight was mostly (95\%) installed in participants' bedrooms. Two participants preferred installation in the hallway, and 1 opted for the staircase.

\section{Outcome Measures}

\section{Primary Outcome Measure}

The mean single-item overnight FOF decreased significantly from $5.5 \pm 3.0$ at baseline to $3.8 \pm 3.2$ at the end of the study $(z=-3.31 ; p=0.001 ; r=0.30)$. The PP analysis yielded similar results, showing a decrease from $5.6 \pm 3.1$ at baseline to $4.0 \pm 3.4$ at the final evaluation $(z=-2.41 ; p=0.016 ; r=0.26)$.

\section{Sleep Quality}

With a significant increase from $6.7 \pm 2.4$ at baseline to $7.4 \pm 1.7$ at the end of the study $(z=-2.52 ; p=0.012$; $r=0.23$ ), the mean single-item sleep quality improved. This increase was not significant in the PP analysis but showed a trend to effect (from $7.0 \pm 2.2$ to $7.5 \pm 1.8$; $z=-1.87 ; p=0.062$ ). 
Fall Rate

At baseline, 14 participants reported fall incidents whilst getting up from bed at night in the previous 6 months (cumulative period of 384 months), resulting in a total of 46 falls. During the study (cumulative period of 225 months), 14 participants reported a total of 25 falls at nighttime. Only 1 participant reported falls before as well as during the study. The mean falls/month fraction during the 6 months before the start of the study did not differ significantly from the falls/month fraction during the study ( $0.1 \pm 0.3$ to $0.1 \pm 0.3 ; z=-0,26 ; p=0,798)$, neither did the fall incidence before and during the study $\left(\chi^{2}[1, n=58]=2.06, p=0.151\right)$.

\section{FES-I, PSQI, and TOPICS-CEP}

The PP analysis showed a significant improvement in mean FES-I scores from $36.9 \pm 12.9$ at baseline to $31.5 \pm$ 11.8 at the final evaluation $(z=-2.69 ; p=0.007)$. Mean PSQI scores, however, did not show a significant change between baseline $(7.0 \pm 3.7)$ and the final evaluation $(6.9 \pm$ 3.8) $(z=-0.65 ; p=0.513)$. TOPICS-CEP improved significantly from $7.0 \pm 1.2$ at baseline to $7.3 \pm 1.0$ at the final evaluation $(t[42]=-3.07 ; p=0.004)$. Results of the ITT and PP analyses are shown in Tables 2 and 3, respectively.

\section{Intervals}

Table 4 describes the development of the mean singleitem FOF overnight and sleep quality during the study in 30 -day intervals. The FOF intervals that significantly differed from each other were: $\mathrm{T}(0)$ compared to $\mathrm{T}(30-59)$ $(z=-3.94, p<0.000), \mathrm{T}(0)$ compared to $\mathrm{T}(60-89)(z=$ $-3.30 ; p=0.001), \mathrm{T}(0)$ compared to $\mathrm{T}(90-119)(z=-2.79$; $p=0.005)$, and $\mathrm{T}(90-119)$ compared to $\mathrm{T}(150-179)(z=$ $-2,42, p=0.016)$. For sleep quality, only $\mathrm{T}(0)$ compared to $\mathrm{T}(180-209)$ was significantly different $(z=-2.21 ; p=$ $0.027)$.

\section{Correlations}

There was a positive correlation between lowering of single-item FOF overnight between the baseline and the final evaluation $(\triangle \mathrm{FOF}=$ primary outcome by LOCF baseline) and time of Gight use $(r=0.306 ; p=0.010)$, and a negative correlation between lowering of single-item FOF overnight and FOF at baseline $(r=-0.581 ; p<0.000)$. There was a positive correlation between single-item FOF at baseline and frailty index $(r=0.323 ; p=0.007)$.

Increase in single-item sleep quality between the baseline and the final evaluation ( $\Delta$ sleep quality = sleep quality by LOCF - baseline) was positively correlated with frailty index $(r=0.307 ; p=0.009)$ and negatively with
Table 1. Demographic variables of participants receiving Gight ${ }^{\mathrm{TM}}$

\begin{tabular}{|c|c|}
\hline Variable & Total $(n=64)$ \\
\hline Female sex & $42(66)$ \\
\hline Age at study entry, years & $81 \pm 8$ \\
\hline \multicolumn{2}{|l|}{ Marital status } \\
\hline Married or long-term cohabitation & $16(25)$ \\
\hline Partner deceased & $39(61)$ \\
\hline Unmarried/divorced & $9(14)$ \\
\hline Dutch origin & $63(98)$ \\
\hline \multicolumn{2}{|l|}{ Living situation } \\
\hline Independent residence, alone & $42(66)$ \\
\hline Independent residence, with others & $15(23)$ \\
\hline Retirement or nursing home & $7(11)$ \\
\hline \multicolumn{2}{|l|}{ Education level } \\
\hline Primary school or less & $13(20)$ \\
\hline Practical/secondary vocational school & $46(72)$ \\
\hline University or tertiary education & $5(8)$ \\
\hline Frailty index & $0.29 \pm 0.13$ \\
\hline \multicolumn{2}{|l|}{ Morbidity (in the last 12 months) } \\
\hline$\geq 2$ diseases & $53(83)$ \\
\hline$\geq 3$ diseases & $38(59)$ \\
\hline Diabetes & $18(28)$ \\
\hline Heart failure & $11(17)$ \\
\hline Stroke or TIA & $7(11)$ \\
\hline Dizziness with falling & $15(23)$ \\
\hline \multicolumn{2}{|l|}{ Care use } \\
\hline Hospital admission (previous 12 months) & $13(20)$ \\
\hline Home care & $36(56)$ \\
\hline Hours home care/week & $5.9 \pm 6.1$ \\
\hline \multicolumn{2}{|l|}{ Problems with walking } \\
\hline None & $13(20)$ \\
\hline Some problems & $50(78)$ \\
\hline Bedridden & $1(2)$ \\
\hline Require help with walking & $31(48)$ \\
\hline Quality of life & $7.3 \pm 1.4$ \\
\hline Very good & $10(16)$ \\
\hline Good & $39(61)$ \\
\hline Reasonable & $14(22)$ \\
\hline Poor & $1(2)$ \\
\hline
\end{tabular}

Numbers (\%) and means \pm SD are shown.

sleep quality at baseline $(r=-0.650 ; p<0.000)$. Sleep quality at baseline was also negatively correlated with frailty index $(r=-0.312 ; p=0.008)$.

\section{Multivariate Regression Analyses}

In a multiple linear regression analysis for change in single-item FOF overnight, a significant result was obtained $(\mathrm{F}[4.53)]=9.392 ; p<0.000)$, with an adjusted $R^{2}$ of 0.371 . Significant predictors for change in overnight FOF were FOF at baseline $(p<0.000)$ and time of Gight use $(p=$ 0.041 ). Participants' difference in FOF decreased 0.76 points 
Table 2. Intention-to-treat analysis for single-item overnight fear of falling (FOF) and sleep quality

\begin{tabular}{lccccc}
\hline Variable & Baseline & LOCF & $p$ value & $z$ value & $\begin{array}{c}\text { Effect size } \\
(r=z / \sqrt{N})\end{array}$ \\
\hline Single-item overnight FOF & $5.5 \pm 3.0$ & $3.8 \pm 3.2$ & 0.001 & -3.31 & -0.30 \\
Single-item sleep quality & $6.7 \pm 2.4$ & $7.4 \pm 1.7$ & 0.012 & -2.52 & -0.23 \\
\hline
\end{tabular}

Means \pm SD. LOCF, last observation carried forward.

Table 3. Per-protocol analysis for primary and secondary outcomes

\begin{tabular}{lcccc}
\hline Variable & Baseline & Final evaluation & $p$ value & $z$ value \\
\hline Single-item overnight fear of falling & $5.6 \pm 3.1$ & $4.0 \pm 3.4$ & 0.016 & -2.41 \\
Single-item sleep quality & $7.0 \pm 2.2$ & $7.5 \pm 1.8$ & 0.062 & -1.87 \\
FES-I & $36.9 \pm 12.9$ & $31.5 \pm 11.8$ & 0.007 & -2.69 \\
TOPICS-CEP & $7.0 \pm 1.2$ & $7.3 \pm 1.0$ & 0.004 & $-3.07^{*}$ \\
PSQI & $7.0 \pm 3.7$ & $6.9 \pm 3.8$ & 0.513 & -0.65 \\
\hline
\end{tabular}

Means \pm SD. Since the Older Persons and Informal Caregivers Survey Minimum Dataset-Composite Endpoint (TOPICS-CEP) scores were distributed normally, a paired $t$ test was performed; therefore, the last column presents a $t$ value. FES-I, international version of the Falls Efficacy Scale; PSQI, Pittsburgh Sleep Quality Inventory.

Table 4. Development of mean overnight fear of falling (FOF) and sleep quality during the study period

\begin{tabular}{llllll}
\hline Interval (days) & $n$ & Mean overnight FOF & SE & Mean sleep quality & SE \\
\hline T (0) & 64 & 5.5 & 0.38 & 6.7 & 0.30 \\
T (0-29) & 18 & 5.1 & 0.57 & 7.2 & 0.50 \\
T (30-59) & 49 & 3.7 & 0.43 & 7.0 & 0.25 \\
T (60-89) & 38 & 3.7 & 0.45 & 7.2 & 0.30 \\
T (90-119) & 31 & 3.4 & 0.41 & 7.2 & 0.26 \\
T (120-149) & 21 & 4.4 & 0.49 & 7.5 & 0.41 \\
T (150-179) & 14 & 5.2 & 0.90 & 7.7 & 0.38 \\
T (180-209) & 11 & 5.1 & 0.98 & 7.5 & 0.51 \\
T (210-239) & 3 & 7.7 & 2.33 & 8.3 & 0.88 \\
\hline
\end{tabular}

for each point of FOF higher at baseline and increased 0.02 points for each day of Gight use. Also, for single-item sleep quality, a significant result was obtained $(\mathrm{F}[4.53]=10.563$; $p<0.000$ ), with an adjusted $R^{2}$ of 0.402 . The only significant predictor of improved sleep quality was sleep quality at baseline $(p<0.000)$, causing an increase in sleep quality with 0.5 points for every point of lower sleep quality at baseline. Regression coefficients are summarized in Table 5.

Qualitative Results

A total of 30 interviews $(68 \%$ out of 44 participants completing the study) was held until saturation was achieved. Demographic variables of interviewees were similar to the demographics of the total study population (age $=80 \pm 8$ years; $73 \%$ female; study length of $128 \pm 53$ days; $77 \%$ living independently alone, $17 \%$ independently with others, and $7 \%$ in retirement or nursing home).

When asked to rate Gight on a scale from 0 (worthless) to 10 (very valuable) interviewees' mean evaluation was $8.4 \pm 0.8$. Seventeen $(57 \%)$, interviewees indicated that Gight had a positive effect on their FOF, stating that it (i) improved their vision or sight $(n=5)$, (ii) prevented falls and/or bumping into objects $(n=4)$, and/or (iii) decreased $(n=5)$ or eliminated $(n=4)$ their fears. The remaining 13 
Table 5. Regression coefficients of change in single-item overnight fear of falling (FOF) and sleep quality between baseline and final outcome (i.e., last observation carried forward)

\begin{tabular}{|c|c|c|c|c|c|c|c|c|}
\hline \multirow[t]{3}{*}{ Model } & \multicolumn{4}{|c|}{ Single-item overnight FOF } & \multicolumn{4}{|c|}{ Single-item sleep quality } \\
\hline & \multicolumn{2}{|c|}{$\begin{array}{l}\text { unstandardized } \\
\text { coefficients }\end{array}$} & \multirow[t]{2}{*}{$\mathrm{T}$} & \multirow[t]{2}{*}{ Sig } & \multicolumn{2}{|c|}{$\begin{array}{l}\text { unstandardized } \\
\text { coefficients }\end{array}$} & \multirow[t]{2}{*}{$\mathrm{T}$} & \multirow[t]{2}{*}{ Sig } \\
\hline & $\mathrm{B}$ & SE & & & $\mathrm{B}$ & SE & & \\
\hline Constant & -0.763 & 1.805 & -0.422 & 0.674 & 3.192 & 0.932 & 3.426 & 0.001 \\
\hline Baseline measurement & -0.758 & 0.147 & -5.166 & 0.000 & -0.499 & 0.088 & -5.685 & 0.000 \\
\hline Living situation & 0.158 & 1.114 & 0.142 & 0.888 & 0.052 & 0.483 & 0.107 & 0.915 \\
\hline Frailty index & 4.190 & 3.550 & 1.181 & 0.243 & 1.361 & 1.546 & 0.880 & 0.383 \\
\hline Time of Gight ${ }^{\mathrm{TM}}$ use & 0.016 & 0.007 & 2.090 & 0.041 & 0.003 & 0.003 & 0.943 & 0.350 \\
\hline
\end{tabular}

(43\%) interviewees did not experience an effect of Gight on their FOF, stating that they (i) had never experienced fear before $(n=8)$, (ii) were still equally afraid $(n=2)$, (iii) did not feel that light influenced their FOF $(n=2)$, or (iv) already had sufficient lighting $(n=1)$. Additional qualitative data are available from the corresponding author (Marcel G.M. Olde Rikkert) upon reasonable request.

\section{Discussion}

\section{Major Findings}

Mean overnight anxiety for falls decreased significantly during the study as well as overall FOF. Also, a significant increase in mean single-item sleep quality was observed, though in the overall PSQI score no significant difference was observed.

\section{Fear of Falling}

FOF is a relevant health risk to older people. It has, for instance, been associated with a greater risk of falling [18, 19], avoidance of activity [20], admittance to care facilities [19], and a decreased capacity to perform daily activities [19]. A randomized controlled study investigating an automated lighting intervention similar to Gight in combination with a tele-assistance service in a group of frail older people reported a lower rate of functional decline within their intervention group [21]. In combination with the results of our study, this finding makes it likely that automated lighting interventions can prevent functional decline by lowering FOF.

\section{Sleep Quality}

Poor sleep quality has also been described as a risk factor for falls $[22,23]$. We hypothesized that Gight could im- prove sleep quality by obviating the need of overhead lighting [24] and by reducing fear at night. In our study, the positive effect of Gight on sleep quality was significant or showed a trend to positive effects in the single-item assessment. However, in the total PSQI, we saw no effect. This may be related to the fact that the single-item question was asked more frequently, causing greater familiarity with it. On the other hand, the results of this study showed no evidence that Gight might cause poor sleep quality, as lighting at night could also cause sleep disruptions [25].

\section{Fall Rate}

The fall rate in this study did not significantly improve since it was too low to determine if Gight had a direct fallpreventive effect. We hypothesized that the lighting provided by Gight during ambulation would have this effect by improving postural stability and spatial awareness [24]. Tchalla et al. [26, 27] conducted randomized controlled trials concerning the aforementioned combined intervention of light paths and tele-assistance services. They demonstrated decreased fall rates in the intervention groups in both a population of frail older people and a population of people with Alzheimer's disease. This indicates that there is a possibility that automated LED pathways prevent falls in different populations, even if not apparent in our study.

\section{Qualitative Evaluation}

The qualitative results of the study showed that participants highly appreciated Gight, as the mean rating of Gight was 8.4 out of 10 points. Since Faes et al. [28] observed that many geriatric patients do not believe that anything can abate their FOF, this additional qualitative evaluation was useful to confirm that the quantitatively observed improvement in FOF was also experienced by participants themselves. The qualitative assessment 
showed that more than half (57\%) of the interviewed participants experienced a subjective improvement in their overnight FOF, thereby confirming the quantitatively measured decrease in FOF.

\section{Long-Term Effects}

The regression analysis for change in overnight singleitem FOF suggests that improvement in FOF decreased linearly with longer application of Gight. Similarly, 1 late interval of the single-item FOF showed a significantly higher FOF than an earlier interval. These results raise the question whether the effect of the lighting intervention reduces over time. An alternative explanation could be that participants with greater overnight FOF were more inclined to remain in the study than participants with milder fears. Since our study cannot sufficiently explain these results, we would recommend the evaluation of the long-term effects of lighting interventions in future research.

\section{Limitations and Strengths}

This study has a few limitations: the most important being the uncontrolled nature of our design. Uncontrolled before-after designs are not suited to account for regression to the mean and confounding factors and are therefore vulnerable to placebo and Hawthorne effects. This limits the certainty to which we can ascribe our results to the use of Gight and often leads to an overestimation of the measured effect [29]. However, the fact that the primary and secondary outcome measures indicate a similar, positive effect strengthens the association of these effects with Gight. As is often the case in research on falls, we had to rely on participants' subjective self-reported data. This meant that we had to rely on questionnaires for FOF and sleep quality and on 6-month recall at baseline and 1 -month recall during the study for fall rate. Additional objective data would have increased the accuracy of our results; however, as the questionnaires used are well validated within our population, the results are valid and generally judged clinically relevant $[30,31]$. As for fall rate, our measurement at baseline is probably subject to recall bias. We tried to improve the reliability of the self-reported fall rate during the study by providing fall calendars to minimize the necessity of recall and by collecting these calendars approximately every month to remind participants to keep recording their falls. Another limitation of this study is its inability to demonstrate an effect on fall rate, which is necessary to determine if Gight is effective in fall prevention. On the other hand, FOF and sleep quality are, as argued above, relevant outcomes for older people, and as both are risk factors for falls, they might indi-

Evaluation Study of a Guiding Nightlight for Older People cate indirect fall-preventive properties of Gight. The qualitative evaluation of participants' experience of Gight is limited by the fact that it was held at the end of our study. Therefore, the experiences of participants that were lost to follow-up or withdrew themselves from the study could not be included. Lastly, our participants were not at high risk of falling, since the fall rate at baseline was relatively close to the average fall rate for people $>65$ years of age, and the fear of falling was only 5.5 on a scale of 10 . We suspect that Gight could have a greater effect on people with a greater risk and fear of falling.

This study is strengthened by the triangulation of quantitative and qualitative data to verify the quantitative results on FOF with participants' subjective experience. This shows that the improvement measured was also of relevance to the participants. Finally, the biggest strength of this study is the simplicity of the intervention, consisting only of a LED strip combined with a motion sensor. This study shows the potential of simple automated lighting interventions in fall prevention, which has been only studied to a limited extent $[21,24,26,27]$.

\section{Further Course of Action and Areas of Further \\ Research}

Further research should be aimed at evaluating the (long-term) effect of automated lighting interventions on fall rates in randomized controlled trials supported by objective data to determine its fall-preventive qualities. This research should also be aimed at comparing different lighting interventions with each other to identify the intervention with the greatest fall-preventive properties, user satisfaction, and cost-effectiveness. The different examples of lighting interventions that have been studied in the past are: (i) unilateral light paths similar to Gight [21, 26, 27], (ii) LED strips positioned on and around objects and doors to provide spatial lighting cues $[24,32]$, and (iii) bilateral pathway demarcation with laser lines [33]. Additionally, more awareness should be raised to the importance of appropriate lighting during nightly ambulation, as older people are often unaware of insufficient lighting [34].

\section{Conclusion}

The lighting intervention Gight shows promising results on overnight FOF and to a lesser extent on sleep quality by automatically illuminating the path from bed to bathroom, but it should be further evaluated before widespread implementation, especially with regard to fall rate and cost-effectiveness. 


\section{Acknowledgment}

The authors wish to thank all students who supported this study: Robert van de Ven, Joris Olde Rikkert, Jay de Jong, Isabel Vos, Jitske Hulzenbos, Shannon Cardol, Wisse van Lamoen, Joris Westerveld, Susanne van Ewijl, Luigi Hoff, Tony Vermeeren, and Mees van Steen. Special thanks go out to our contact persons at BrabantZorg, Frank van Putten, and Kathleen Westhoff, and at the municipality of Oss, Leontien van Hassel. Thessa W. Thölking is a medical student participating in the Honours Program of the Faculty of Medicine, University Medical Center Utrecht.

\section{Statement of Ethics}

The Ethical Committee of the Radboudumc (Nijmegen, NL) approved the study protocol. Voluntary participation was documented in written informed consents, as well as the participants' right to withdraw themselves from the study at any time. All data were stored and analyzed anonymously. The protocol was registered in the Dutch trial database (No. NL6938).

\section{Disclosure Statement}

Ms. Thölking received a reimbursement of expenses from Gight-BV. Mr. Lamers held stocks in Gight-BV until April 2019.

\section{Funding Sources}

This work was equally supported by BrabantZorg (an elderly care organization) and Oss Innoveert (the municipality of Oss) covering personnel and material costs through funding. Both parties were not involved in the design, data collection, analysis, and reporting of the study.

\section{Author Contributions}

All authors contributed to the design of the study. T.W.T. and E.C.T.L. planned and supervised data collection. T.W.T. and M.G.M.O.R. performed data analysis and drafted the manuscript. All authors discussed the results and commented on the manuscript.

\section{References}

1 Stam C. Privé-ongevallen bij ouderen: Ongevalscijfers 2017 [Internet]. VeiligheidNL; 2018 [cited 2019 Feb 23]. Available from: www. google.com/url? $\mathrm{sa}=\mathrm{t} \& \mathrm{rct}=\mathrm{j} \& \mathrm{q}=\& \mathrm{esrc}=\mathrm{s} \&$ sour $\mathrm{ce}=$ web $\& \mathrm{~cd}=2 \& \mathrm{cad}=\mathrm{rja} \&$ uact $=8 \& \mathrm{ved}=2 \mathrm{ahU}$ KEwiSjrLasMviAhWHaVAKHZyRDokQFjA BegQIARAC\&url=https\%3A\%2F\%2Fwww. veiligheid.nl\%2F.ibmmodres\%2Fdomino\%2F OpenAttachment\%2FVeiligheid\%2FWebsite. nsf\%2F12089CE60C2D7769C12583AC00544 $425 \% 2$ Fasset $\% 2$ FCijferrapportage $\% 2520$ Valo ngevallen\%2520ouderen\%25202017.pdf\& usg=AOvVaw33JP57sZWW7HKdfrpcKWPz.

2 Tromp AM, Pluijm SM, Smit JH, Deeg DJ, Bouter LM, Lips P. Fall-risk screening test: a prospective study on predictors for falls in community-dwelling elderly. J Clin Epidemiol. 2001 Aug;54(8):837-44.

3 Brown MJ, Jacobs DE. Residential light and risk for depression and falls: results from the LARES study of eight European cities. Public Health Rep. 2011 May-Jun;126 Suppl 1:13140.

4 Connell BR, Wolf SL; Atlanta FICSIT Group. Environmental and behavioral circumstances associated with falls at home among healthy elderly individuals. Arch Phys Med Rehabil. 1997 Feb;78(2):179-86.

5 Mazharizad F, Dadipoor S, Madani A, Moradabadi AS. Investigating the prevalence and causes of events leading to falls among the elderly hospitalized in Bandar Abbas hospitals. J Educ Health Promot. 2015 Feb;4:11.

6 Magota C, Sawatari H, Ando SI, Nishizaka MK, Tanaka K, Horikoshi K, et al. Seasonal ambient changes influence inpatient falls. Age Ageing. 2017 May;46(3):513-7.
7 Salonen L, Kivelä SL. Eye diseases and impaired vision as possible risk factors for recurrent falls in the aged: a systematic review. Curr Gerontol Geriatr Res. 2012;2012:271481.

8 Bouma H, Weale RA, McCreadie C. Technological environments for visual independence in later years. Gerontechnology (Valkenswaard). 2006;5(4):187-94.

9 Brooke-Wavell K, Perrett LK, Howarth PA, Haslam RA. Influence of the visual environment on the postural stability in healthy older women. Gerontology. 2002 Sep-Oct;48(5): 293-7.

10 Stewart RB, Moore MT, May FE, Marks RG, Hale WE. Nocturia: a risk factor for falls in the elderly. J Am Geriatr Soc. 1992 Dec;40(12): 1217-20.

11 Bosch JL, Weiss JP. The prevalence and causes of nocturia. J Urol. 2010 Aug;184(2):440-6.

12 Dewan N, MacDermid JC. Fall Efficacy ScaleInternational (FES-I). J Physiother. 2014 Mar; 60(1):60.

13 Buysse DJ, Reynolds CF 3rd, Monk TH, Hoch CC, Yeager AL, Kupfer DJ. Quantification of subjective sleep quality in healthy elderly men and women using the Pittsburgh Sleep Quality Index (PSQI). Sleep. 1991 Aug;14(4):331-8.

14 Lutomski JE, Baars MA, Schalk BW, Boter H, Buurman BM, den Elzen WP, et al.; TOPICSMDS Consortium. The development of the Older Persons and Informal Caregivers Survey Minimum DataSet (TOPICS-MDS): a large-scale data sharing initiative. PLoS One. 2013 Dec;8(12):e81673.

15 Hofman CS, Makai P, Boter H, Buurman BM, de Craen AJ, Olde Rikkert MG, et al. Establishing a composite endpoint for measuring the effectiveness of geriatric interventions based on older persons' and informal caregivers' preference weights: a vignette study. BMC Geriatr. 2014 Apr;14(51):51.

16 Searle SD, Mitnitski A, Gahbauer EA, Gill TM, Rockwood K. A standard procedure for creating a frailty index. BMC Geriatr. 2008 Sep;8(24):24.

17 Lutomski JE, Baars MA, van Kempen JA, Buurman BM, den Elzen WP, Jansen AP, et al. Validation of a frailty index from the older persons and informal caregivers survey minimum data set. J Am Geriatr Soc. 2013 Sep; 61(9):1625-7.

18 Friedman SM, Munoz B, West SK, Rubin GS, Fried LP. Falls and fear of falling: which comes first? A longitudinal prediction model suggests strategies for primary and secondary prevention. J Am Geriatr Soc. 2002 Aug; 50(8):1329-35.

19 Cumming RG, Salkeld G, Thomas M, Szonyi G. Prospective study of the impact of fear of falling on activities of daily living, SF-36 scores, and nursing home admission. J Gerontol A Biol Sci Med Sci. 2000 May;55(5):M299-305.

20 Zijlstra GA, van Haastregt JC, van Eijk JT, van Rossum E, Stalenhoef PA, Kempen GI. Prevalence and correlates of fear of falling, and associated avoidance of activity in the general population of community-living older people. Age Ageing. 2007 May;36(3):304-9.

21 Lachal F, Tchalla AE, Cardinaud N, Saulnier I, Nessighaoui H, Laubarie-Mouret C, et al. Effectiveness of light paths coupled with personal emergency response systems in preventing functional decline among the elderly. SAGE Open Med. 2016 Aug;4:2050312116665764. 
22 St George RJ, Delbaere K, Williams P, Lord SR. Sleep quality and falls in older people living in self- and assisted-care villages. Gerontology. 2009;55(2):162-8.

23 Latimer Hill E, Cumming RG, Lewis R, Carrington S, Le Couteur DG. Sleep disturbances and falls in older people. J Gerontol A Biol Sci Med Sci. 2007 Jan;62(1):62-6.

24 Chari SR, Smith S, Mudge A, Black AA, Figueiro M, Ahmed M, et al. Feasibility of a stepped wedge cluster RCT and concurrent observational sub-study to evaluate the effects of modified ward night lighting on inpatient fall rates and sleep quality: a protocol for a pilot trial. Pilot Feasibility Stud. 2016 Jan; 2(1): 1 .

25 Figueiro MG. A proposed $24 \mathrm{~h}$ lighting scheme for older adults. Light Res Technol. 2008;40(2):153-60.

26 Tchalla AE, Lachal F, Cardinaud N, Saulnier I, Bhalla D, Roquejoffre A, et al. Efficacy of simple home-based technologies combined with a monitoring assistive center in decreasing falls in a frail elderly population (results of the Esoppe study). Arch Gerontol Geriatr. 2012 Nov-Dec;55(3):683-9.

27 Tchalla AE, Lachal F, Cardinaud N, Saulnier I, Rialle V, Preux PM, et al. Preventing and managing indoor falls with home-based technologies in mild and moderate Alzheimer's disease patients: pilot study in a community dwelling. Dement Geriatr Cogn Disord. 2013; 36(3-4):251-61.

28 Faes MC, Reelick MF, Joosten-Weyn Banningh LW, Gier M, Esselink RA, Olde Rikkert MG. Qualitative study on the impact of falling in frail older persons and family caregivers: foundations for an intervention to prevent falls. Aging Ment Health. 2010 Sep; 14(7): $834-42$.

29 Grimshaw J, Campbell M, Eccles M, Steen N. Experimental and quasi-experimental designs for evaluating guideline implementation strategies. Fam Pract. 2000 Feb; 17(90001 Suppl 1):S11-6.

30 Delbaere K, Close JC, Mikolaizak AS, Sachdev PS, Brodaty H, Lord SR. The Falls Efficacy Scale International (FES-I). A comprehensive longitudinal validation study. Age Ageing. 2010 Mar;39(2):210-6.

31 Mollayeva T, Thurairajah P, Burton K, Mollayeva S, Shapiro CM, Colantonio A. The Pittsburgh sleep quality index as a screening tool for sleep dysfunction in clinical and nonclinical samples: A systematic review and meta-analysis. Sleep Med Rev. 2016 Feb;25:5273.

32 Figueiro MG, Gras LZ, Rea MS, Plitnick B, Rea MS. Lighting for improving balance in older adults with and without risk for falls. Age Ageing. 2012 May;41(3):392-5.

33 Figueiro MG, Plitnick B, Rea MS, Gras LZ, Rea MS. Lighting and perceptual cues: effects on gait measures of older adults at high and low risk for falls. BMC Geriatr. 2011 Aug; 11(1):49.

34 Kamei T, Kajii F, Yamamoto Y, Irie Y, Kozakai R, Sugimoto T, et al. Effectiveness of a home hazard modification program for reducing falls in urban community-dwelling older adults: A randomized controlled trial Jpn J Nurs Sci. 2015 Jul;12(3):184-97. 